\title{
Correction to: Psychiatric genetics and the structure of psychopathology
}

\author{
Jordan W. Smoller ${ }^{1,2,3} \cdot$ Ole A. Andreassen $\mathbb{1}^{4,5} \cdot$ Howard J. Edenberg $\mathbb{C}^{6} \cdot$ Stephen V. Faraone $\mathbb{C}^{7} \cdot$ Stephen J. Glatt $^{7}$. \\ Kenneth S. Kendler ${ }^{8,9}$
}

Published online: 14 March 2018

(c) Macmillan Publishers Limited, part of Springer Nature 2018

Correction to: Molecular Psychiatry (2018); https://doi. org/10.1038/s41380-017-0010-4.

In the Table 1 legend, the reference numbers and symbols were not correctly presented in the footnotes. The corrected footnotes are presented below.

On the diagonal: Heritability estimates (and 95\% CI) from pedigree/twin studies (lower, shaded orange) and genomewide SNP analyses (upper, shaded light blue). Also pairwise genetic correlations from pedigree/twin studies (below diagonal) and genomewide SNP analyses (above diagonal) are shown. SNP-based estimates (light blue) are taken from ref. 77. Bolded entries: 95\% CI does not include 0.0 .

Sources: * [99], ¥ [100], \& [101],! [102], \$ [103], II [104], \% [105], @ [106], $\phi[107], \S[108], \sqrt{ }$ [109]

$A S D$ autism spectrum disorder, $A D H D$ attention deficithyperactivity disorder, $S C Z$ schizophrenia, $B D$ bipolar disorder, $M D D$ major depressive disorder, $A N$ anorexia nervosa, $O C D$ obsessive-compulsive disorder, $P T S D$ posttraumatic stress disorder, N/A not available

The original article has been updated.
The original article can be found online at https://doi.org/10.1038/ s41380-017-0010-4.

Jordan W. Smoller

jsmoller@mgh.harvard.edu

1 Psychiatric and Neurodevelopmental Genetics Unit, Center for Genomic Medicine, Massachusetts General Hospital, Boston, MA, USA

2 Department of Psychiatry, Massachusetts General Hospital, Boston, MA, USA

3 Stanley Center for Psychiatric Research, Broad Institute of MIT and Harvard, Cambridge, MA, USA

4 NORMENT_KG Jebsen Centre, University of Oslo, Oslo, Norway
5 Division of Mental Health and Addiction, Oslo University Hospital, Oslo, Norway

6 Department of Biochemistry and Molecular Biology, Indiana University School of Medicine, Indianapolis, IN, USA

7 Departments of Psychiatry and of Neuroscience and Physiology, SUNY Upstate Medical University, Syracuse, NY, USA

8 Virginia Institute for Psychiatric and Behavioral Genetics, Virginia Commonwealth University, Richmond, VA, USA

9 Department of Psychiatry, Virginia Commonwealth University, Richmond, VA, USA 